\title{
Low-level Cxcr4-haploinsufficient HSC engraftment is sufficient to correct leukopenia in WHIM syndrome mice
}

\author{
Ji-Liang Gao, ${ }^{1}$ Albert Owusu-Ansah, ${ }^{1}$ Andrea Paun, ${ }^{2}$ Kimberly Beacht, ${ }^{2}$ Erin Yim, ${ }^{1}$ Marie Siwicki, \\ Alexander Yang, ' Qian Liu, 'David H. McDermott,' and Philip M. Murphy'
}

${ }^{1}$ Molecular Signaling Section, Laboratory of Molecular Immunology, and 'Intracellular Parasite Biology Section, Laboratory of Parasitic Diseases, National Institute of Allergy and Infectious Diseases, NIH, Bethesda, Maryland, USA.

\begin{abstract}
Warts, hypogammaglobulinemia, infections, and myelokathexis (WHIM) syndrome immunodeficiency is caused by autosomal dominant gain-of-function mutations in chemokine receptor CXCR4. Patient WHIM-09 was spontaneously cured by chromothriptic deletion of 1 copy of 164 genes, including the CXCR $4^{\text {WHIM }}$ allele, presumably in a single hematopoietic stem cell (HSC) that repopulated HSCs and the myeloid lineage. Testing the specific contribution of CXCR4 hemizygosity to her cure, we previously demonstrated enhanced engraftment of $C x C r 4^{+/ 0}$ HSCs after transplantation in WHIM ( $\left.\mathrm{CxCr}^{+/ w}\right)$ model mice, but the potency was not quantitated. We now report graded-dose competitive transplantation experiments using lethally irradiated $\mathrm{Cxcr}^{+/+}$recipients in which mixed BM cells containing approximately $5 \mathrm{Cxcr} 4^{+/ 0} \mathrm{HSCs}$ and a 100 -fold excess of $\mathrm{Cxcr}^{+/ w} \mathrm{HSCs}$ achieved durable $50 \% \mathrm{Cxcr}^{+/ 0}$ myeloid and B cell chimerism in blood and approximately $20 \% \mathrm{Cxcr}^{+/ 0} \mathrm{HSC}$ chimerism in BM. In $\mathrm{Cxcr} 4^{+/ 0} / \mathrm{Cxcr}^{+/ \omega}$ parabiotic mice, we observed $80 \%-100 \% \mathrm{CxCr}^{+/ 0}$ myeloid and lymphoid chimerism in the blood and $15 \% \mathrm{Cxcr}^{+/ 0} \mathrm{HSC}$ chimerism in BM from the $\mathrm{Cxcr}^{+/ \mathrm{w}}$ parabiont, which was durable after separation from the $\mathrm{Cxcr} 4^{+/ 0}$ parabiont. Thus, CXCR4 haploinsufficiency likely significantly contributed to the selective repopulation of HSCs and the myeloid lineage from a single chromothriptic HSC in WHIM-09. Moreover, the results suggest that WHIM allele silencing of patient HSCs is a viable gene therapy strategy.
\end{abstract}

Conflict of interest: JLG, MS, EY, QL, DHM, and PMM are listed as inventors on United States patent application 20170196911

Copyright: (c) 2019, American Society for Clinical Investigation.

Submitted: July 28, 2019

Accepted: October 29, 2019

Published: December 19, 2019

Reference information: /CI Insight. 2019;4(24):e132140.

https://doi.org/10.1172/jici.

insight.132140.

\section{Introduction}

Warts, hypogammaglobulinemia, infections, and myelokathexis (WHIM) syndrome is a rare, combined primary immunodeficiency disease caused in almost all cases by autosomal dominant, gain-of-function mutations in the gene encoding chemokine receptor CXCR4 $(1,2)$. In response to its principal agonist CXCL12, CXCR4 signaling normally mediates homing, retention, and multilineage differentiation of hematopoietic stem and progenitor cells in BM niches by regulating their migration, survival, and quiescence (3-6). CXCR4 signaling also normally regulates migration and distribution of most types of mature leukocytes. WHIM mutations, which block normal arrestin-dependent CXCR4 desensitization and downregulation processes, distort normal leukocyte trafficking and distribution, resulting in neutropenia and even panleukopenia in many patients, despite normal hematopoietic development (7-9). Neutropenia is typically severe and occurs despite BM myeloid hyperplasia due to BM retention of fully mature and degenerating neutrophils (10-13). This striking hematopathologic picture, which is almost pathognomonic of WHIM syndrome, is called myelokathexis, a Greek neologism meaning "marrow retention" and the "M" in the acronym WHIM (14).

We previously reported that patient WHIM-09 was cured of WHIM syndrome when the disease allele CXCR $4^{R 334 X}$ and 163 other genes were spontaneously deleted on 1 copy of chromosome 2 by chromothripsis, presumably in a single hematopoietic stem cell (HSC) (15). Chromothripsis refers to random chromosome shattering, resulting in all-at-once complex chromosomal rearrangements and gene deletions and duplications (16). It occurs in a small percentage of cancers and has been reported in a few patients with congenital neurodevelopmental disorders $(17,18)$. WHIM-09 is the only patient known to have had a beneficial outcome from chromothripsis (15). Fortuitously, the original chromothriptic HSC in WHIM-09 acquired a selective advantage and repopulated both the HSC pool and the entire myeloid lineage. The 
absolute neutrophil count and absolute monocyte count rose to new baselines approximately 1.5- to 2-fold above normal limits, where they have remained stable for $>20$ years, associated with both spontaneous clearance of warts and cessation of recurrent acute infections. In contrast to the myeloid lineage, the chromothriptic HSCs were unable to repopulate the patient's lymphoid lineage, so that she became a hematopoietic chimera of WHIM lymphoid cells and chromothriptic non-WHIM myeloid cells hemizygous (genotype +/o) for CXCR4 and the other 163 deleted genes. This implies that the WHIM allele drives major clinical phenotypes of the disorder in a myeloid-dependent manner.

Competitive BM and HSC transplantation of 50:50 mixtures of donor cells from all paired combinations of $\mathrm{CxCr}^{+/ o}, \mathrm{CxCr}^{+/+}$, and $\mathrm{Cxcr} 4^{+/ w}$ ("w" denotes knockin of the human WHIM allele S338X) mice into lethally irradiated congenic $C x c r 4^{+/+}$recipient mice suggested that deletion of the WHIM allele per se might be necessary and sufficient to account for WHIM-09's cure, independently of the other 163 deleted genes (15). In particular, the results of the $C x c r 4^{+/ o} / C x c r 4^{+/ w}$ HSC competition showed $>95 \% C x c r 4^{+/ o}$ myeloid and B cell chimerism in the blood and approximately $80 \% \mathrm{Cxcr} 4^{+/ o}$ hematopoietic stem and progenitor cell and mature leukocyte chimerism in the BM, associated with enhanced proliferation of $C x \mathrm{Cr}^{+/ o} \mathrm{HSCs}$ in vivo. Consistent with this, CXCR4 signaling had previously been shown to suppress HSC proliferation (5), suggesting a cure mechanism for WHIM-09 involving a competitive proliferative advantage in HSCs conferred by $C X C R 4$ haploinsufficiency.

We also reported a competitive advantage for $\mathrm{CxCr}^{+/ o} \mathrm{HSCs}$ over $\mathrm{CxCr4^{+/+ }}$ and $\mathrm{CxCr4^{+/w }}$ HSCs for hematopoietic reconstitution after transplantation into unconditioned congenic $C x \mathrm{Cr} 4^{+/ w}$ recipient mice (19). In this case, competitive transplantation of 50 million $C x c r 4^{+/ o}$ total BM cells ( 5000 HSCs, ref. 20) into unconditioned WHIM recipient mice resulted in approximately $70 \%$ durable $C x c r 4^{+/ o}$ donor $\mathrm{CD} 11 \mathrm{~b}^{+}$ myeloid cell chimerism in the blood, despite achieving only $6 \% \mathrm{Cxcr} 4^{+/ 0}$ donor HSC chimerism in the BM.

Our previous studies did not define the magnitude of the selective advantage conferred by CXCR4 haploinsufficiency on HSC engraftment efficiency, which is important for considerations of gene therapy strategy in the disease $(15,19)$. Moreover, a major difference between transplantation experiments in mice and the spontaneous genetic cure that occurred in WHIM-09, other than the additional 163 genes deleted in WHIM-09, is the manipulation and transplantation of HSCs in the former but not the latter. Here, we address both of these issues by experiments in (a) lethally irradiated recipient mice competitively transplanted with graded ratios of $C x c r 4^{+/ o}$ and $C x c r 4^{+/ w}$ donor BM cells and (b) parabiotic mice, in which HSC manipulation and transplantation are obviated.

\section{Results}

$C X c r 4^{+/ o}$ donor BM cells have a strong selective advantage over CXcr $4^{+/ w}$ donor BM cells for reconstituting myeloid and $B$ cells in the blood after transplantation of lethally irradiated mice. To better understand the role played by CXCR4 haploinsufficiency in the cure of patient WHIM-09, we performed competitive BM transplantation experiments under increasingly stringent conditions, first by transplanting 5 million donor BM cells with different ratios of $\mathrm{CxCr}^{+/ o}$ and $\mathrm{Cxcr} 4^{+/ w}$ cells into lethally irradiated congenic $\mathrm{Cxcr} 4^{+/+}$recipient mice. Our hypothesis was that if CXCR4 haploinsufficiency per se in the original chromothriptic HSC was necessary and sufficient to cure her, then in competitive $\mathrm{CxCr}^{+/ o}$ and $\mathrm{Cxcr} 4^{+/ w} \mathrm{BM}$ cell transplantation experiments in lethally irradiated mice we might observe a threshold dose of $\mathrm{CxCr}^{+/ o}$ donor BM cells for full reconstitution but not a graded $\mathrm{Cxcr}^{+/ o}$ donor BM cell dose response. Donor and recipient mouse hematopoietic cells were differentially marked by CD45 polymorphisms for quantitation of reconstitution by flow cytometry over time (CD45.1 marked $\mathrm{Cxcr}^{+/+}, \mathrm{CD} 45.2$ marked $\mathrm{Cxcr} 4^{+/ w}$, and CD45.1/CD45.2 marked $\mathrm{Cxcr4^{+/o }}$ ). We have previously reported for these donors that the HSC frequencies in total BM are equivalent and that transplantation of purified HSCs gives equivalent results to transplantation of total BM cells when the input cell ratio is 50:50 (15).

Confirming our previous report, transplanting an approximately 50:50 mixture of the $2 \mathrm{BM}$ donors resulted in durable approximately $100 \% \mathrm{Cxcr}^{+/ o}$ donor-derived myeloid and B cell chimerism in the blood $(15,19)$ (Figure 1, A-C). In contrast, as we have previously reported, the posttransplantation donor-derived $\mathrm{T}$ cell ratio in recipient blood was similar to the input value $(15,19)$ (Figure $1 \mathrm{~B})$. The reason for $\mathrm{T}$ cell exceptionalism in this transplantation model is not clear and will require additional focused research. Full $\mathrm{CxCr}^{+/ o}$ donor-derived myeloid and B cell chimerism in the blood was also observed, even when only $25 \%$ of the transplanted BM cells were from the $C x c r 4^{+/ o}$ donor (n.b., this represents approximately 125 HSCs, since approximately $0.01 \%$ of whole BM cells are HSC, ref. 20) (Figure 1B). However, a clear Cxcr4 ${ }^{+/ o}$ 
A

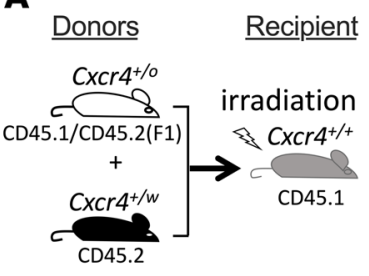

Bone marrow

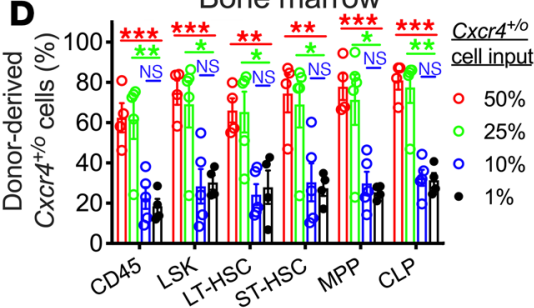

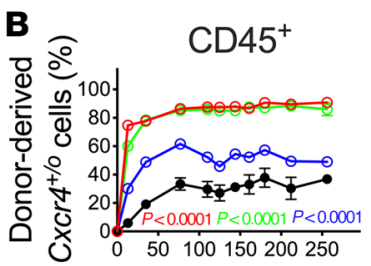
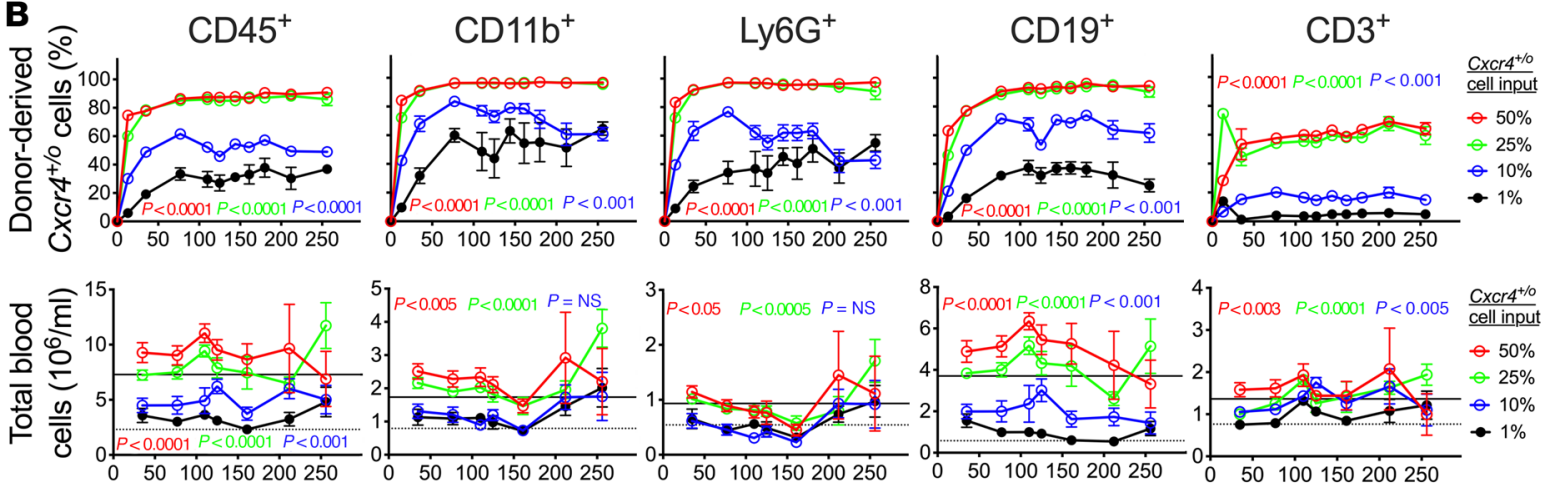

Days post bone marrow transfer
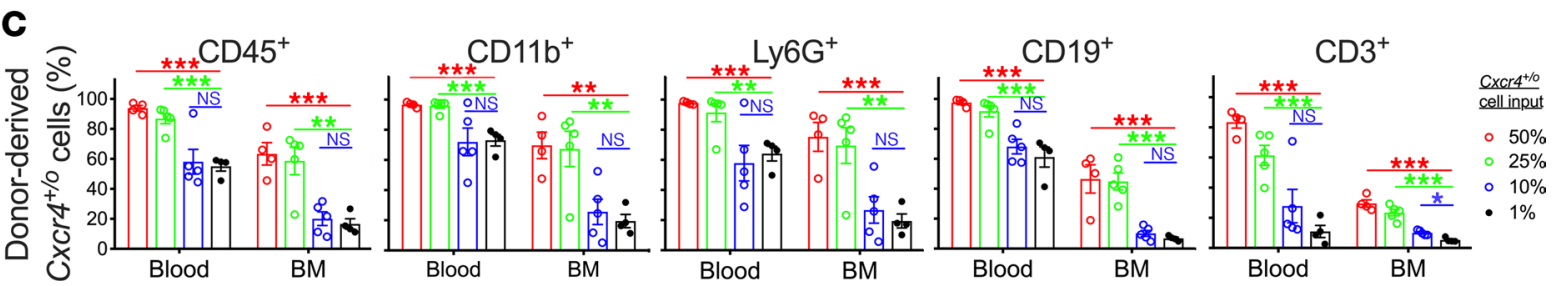

Figure 1. $\mathrm{CxCr}^{+/ 0}$ donor BM cells have a strong selective advantage over $\mathrm{CxCr} 4^{+/ \mathrm{w}}$ donor BM cells for reconstituting myeloid and B cells in the blood of lethally irradiated mice. (A) Experimental design. CxCr4 genotypes and congenic CD45 isoforms are indicated above and below the mouse cartoons, respectively. A total of 5 million total BM cells were transplanted at different donor cell ratios, indicated as the percentage $C x \mathrm{Cr}^{+/ 0}$ input cells on the right of B, C, and D. (B) Time course of hematopoietic reconstitution. Each graph corresponds to the immunophenotypically defined blood cell subset indicated at the top of the column in which it is found. Top row of graphs: $\mathrm{CxCr}_{\mathrm{C}}{ }^{+/ 0}$ donor-derived blood cell chimerism, presented as the percentage (mean $\pm \mathrm{SEM}$ ) of the total cell number for each subset. Bottom row of graphs: total absolute blood counts for each leukocyte subset (mean \pm SEM). Solid lines and dashed lines indicate average values of blood counts for each subset in unmanipulated control $\mathrm{CxCr}^{+/+}(n=58)$ and $C x \mathrm{Cr} 4^{+/ \omega}(n=38)$ mice in our colony. (C and $\left.\mathbf{D}\right)$ Chimerism in BM versus blood for $\mathrm{CxCr}^{+/ 0}$ donor-derived mature leukocytes (C) and HSCs and HPCs in BM (D). The blood data are the same as at day 256 for the top graphs in B, the day when the experiment was terminated for BM analysis. For all conditions, $n=5$ for each group. SEM was less than $5 \%$ of the mean at all time points lacking visible error bars. The experiment was repeated once with a similar pattern. In $\mathbf{B}, P$ values are color coded to refer to the corresponding color-coded $C x \mathrm{Cr}^{+/ 0}$ input percentage result compared with the $1 \%\left[x \mathrm{Cr} 4^{+/ 0}\right.$ input cell result. ${ }^{*} P<0.05 ;{ }^{* *} P<0.01 ;{ }^{* * *} P<0.005$. Single comparisons, Student's $t$ test; multiple comparisons, 2-way ANOVA. LSK, lineage-Sca-1+c-Kit ${ }^{+}$BM cells; LT- and ST-HSC, long-term and short-term hematopoietic stem cells; MPP, multipotential progenitor cells; CLP, common lymphoid precursor cells.

donor $\mathrm{BM}$ cell dose response became apparent at lower ratios. Remarkably, at the lowest ratio tested, $1 \%$ input $\mathrm{Cxcr}^{+/ o}$ donor BM cells ( $\left.\sim 5 \mathrm{HSCs}\right)$ still resulted in over 50\% $\mathrm{Cxcr}^{+/ o}$ donor-derived myeloid and B cell chimerism in the blood (Figure 1B), which was sufficient to stably improve circulating myeloid and B cell levels in the transplanted animals to the normal range. In contrast, and similar to our previous studies, the absolute numbers of $\mathrm{Cxcr}^{+/ w}$ donor-derived myeloid cells and B cells in the blood after competitive transplantation remained below even the leukopenic norm for unmanipulated $\mathrm{Cxcr}^{+/ w}$ mice.

When the mice were sacrificed 256 days after transplantation (a date chosen arbitrarily once durability of chimerism was established), we found that the frequencies of $\mathrm{Cxcr} 4^{+/ o}$ donor-derived myeloid cells and B cells also significantly increased in the BM compared with the input donor cell frequencies across all input donor cell ratios tested, although frequencies increased to a lesser extent than in peripheral blood (Figure 1C). Consistent with this, the frequencies of $\mathrm{Cxcr}^{+/ o}$ donor-derived HSCs and hematopoietic progenitor cells (HPCs) also significantly increased in the BM compared with the input frequencies across all input donor cell ratios tested (Figure 1D). Thus, the predominance of $\mathrm{Cxcr}^{+/ o}$ over $\mathrm{Cxcr}^{+/ w}$ donor-derived leu- 
kocytes in the blood may be the result of both superior engraftment of $\mathrm{Cxcr}^{+/ o} \mathrm{HSCs}$ and superior egress of mature $\mathrm{Cxcr}^{+/ o}$ leukocytes. Nevertheless, the dose-response results do not entirely explain WHIM-09's cure, because there is a limit to the ability of transplanted and engrafted $C x c r 4^{+/ o}$ HSCs to fully reconstitute hematopoiesis when placed in competition with $\mathrm{Cxcr}^{+/ w} \mathrm{HSCs}$ in this conditioned transplantation system.

Cxcr $4^{+/ o}$ leukocyte chimerism predominates in both parabionts of $\mathrm{CxCr}^{+/ o} / \mathrm{Cxcr} 4^{+/ w}$ parabiotic mice. We next used parabiotic pairing of unconditioned $C x c r 4^{+/ w}$ and $C x c r 4^{+/ o}$ mice to test whether the process of harvesting $\mathrm{CxCr}^{+/ o} \mathrm{BM}$ cells for transplantation, which did not happen in WHIM-09, might affect HSC engraftment efficiency. As with the transplantation model, in the parabiosis model, Cxcr4 was the only 1 of the 164 genes deleted in WHIM-09 that was tested for the effects of haploinsufficiency on engraftment and chimerism. In particular, $\mathrm{CxCr}^{+/ 0}$ mice were surgically joined with age- and sex-matched congenic CD45 isoform-marked $\mathrm{CxCr}^{+/ w}$ mice (designated as parabiotic pair $\mathrm{Cxcr} 4^{+/ o} / \mathrm{Cxcr}^{+/ w}$ ) to model the HSC competition that occurred in WHIM-09; however, only competition between Cxcr4 genotypes was studied (Figure 2A). As a control, we generated parabiotic pairs comprising $\mathrm{CxCr}^{+/+}$mice joined to congenic $\mathrm{CD} 45$ isoform-marked $\mathrm{Cxcr} 4^{+/+}$ mice (designated as parabiotic pair $C x \mathrm{Cr}^{+/+} / C x C r 4^{+/+}$) (Figure 2A). This control allowed assessment of the background pattern of leukocyte distribution in peripheral blood and BM established by parabiosis per se. After 5 months of parabiosis (the connected phase), 3 of the 5 pairs of parabiotic mice from both the experimental and control groups were terminated for BM analysis without first being separated. To test whether chimeric HSCs were stably engrafted, we surgically separated the remaining 3 parabiotic pairs (2 in the $\mathrm{CxCr} 4^{+/ o} / \mathrm{CxCr}^{+/ w}$ group and 1 in the $\mathrm{CxCr}^{+/+} / \mathrm{CxCr}^{+/+}$group) 5 months after parabiotic joining and then followed the separated parabionts for an additional 5 months (the separated phase) when they were arbitrarily sacrificed for BM analysis (see Figure 2A for experimental design).

After connecting the control $\mathrm{CxCr}^{+/+} / \mathrm{CxCr}^{+/+}$parabiotic pair, peripheral blood chimerism was established quickly at approximately 30\% for myeloid cells and approximately $45 \%$ for both B and T cells and was sustained at these levels across the entire time course of the connected phase, with no significant differences observed between the parabionts of each parabiotic pair (Figure $2 \mathrm{~B}$, top). The reason the frequencies are not $50 \%$, as would be predicted from simple mixing of the circulations, and differ between different subsets may be explained by the limited circulatory connection in the connected skin, which is made up of only small blood vessels across a limited area of contact, and the different rates of production of different leukocyte subsets (21). At the end of the connected phase, we found no difference between chimerism levels measured in blood and BM for either B cells or T cells ( 45\%), whereas chimerism for myeloid cells was only approximately $5 \%$ in BM compared with approximately $30 \%$ in blood (Figure $2 \mathrm{C}$, top). HSC chimerism in BM from the $\mathrm{Cxcr} 4^{+/+} / \mathrm{CxCr}^{4^{+/}}$parabiotic pairs was even lower at approximately $3 \%$, without significant differences for all subsets in both parabionts (Figure 2E). After separation, mature leukocyte chimerism in the peripheral blood of each $\mathrm{Cxcr}^{+/+}$parabiont fell for each subset to a new stable level. In particular, $\mathrm{CxCr} 4^{+/+}$myeloid chimerism fell quickly from approximately $30 \%$ to approximately $5 \%$, whereas $\mathrm{B}$ and $\mathrm{T}$ cell chimerism both fell much more gradually over 5 months from $45 \%$ to approximately $20 \%$ (Figure 2B, top). The different rates of reduction of chimerism between myeloid and lymphoid cells may be explained by their different half-lives. At the end of the separated phase, BM HSC and HPC chimerism was $3 \%$ for both parabionts (Figure $2 \mathrm{E}$ ), the same as at the end of the connected phase. Although we only tested $1+/+:+/+$ parabiotic pair in the separated phase, the results were similar to previous reports for this phase in wild-type parabiotic mice $(22,23)$.

Compared with this control parabiosis hematopoietic chimerism pattern, chimerism levels in the $\mathrm{Cxcr}^{+/ o} / \mathrm{CxCr}^{+/ w}$ parabiotic pairs were very different. In particular, $\mathrm{Cxcr} 4^{+/ o}$ myeloid cells, $\mathrm{B}$ cells, and $\mathrm{T}$ cells all rapidly reconstituted in the $C x c r 4^{+/ w}$ parabiont after establishment of parabiosis, peaking at approximately day 20 postparabiotic connection at approximately $60 \%-70 \%$ for myeloid cells and approximately $80 \%-90 \%$ for B and T cells. These levels were maintained with limited excursion for 5 months, when the animals were sacrificed or separated. Conversely, $C x c r 4^{+/ w}$ chimerism levels were very low ( $<20 \%$ for each subset) in the $\mathrm{Cxcr}^{+/ o}$ parabionts (Figure 2B, bottom).

At the end of the connected phase, $C x c r 4^{+/ o}$ HSC and HPC chimerism was much higher in BM from the $C x c r 4^{+/ w}$ parabiont than was $C x c r 4^{+/ w}$ HSC and HPC chimerism in BM from the $C x c r 4^{+/ o}$ parabiont $(15 \%-20 \%$ vs. $1 \%-4 \%$, respectively) (Figure $2 \mathrm{E}$ ). Mature $\mathrm{Cxcr} 4^{+/ o}$ myeloid and lymphoid cell chimerism in the $C x \mathrm{Cr} 4^{+/ w}$ parabionts was much lower in BM than in blood, with myeloid chimerism in BM only approximately $15 \%$ and lymphoid chimerism in BM only $35 \%$ and $25 \%$ for B and T cells, respectively (Figure 2C, bottom). Cxcr $4^{+/ w}$ chimerism in BM from the $\mathrm{Cxcr}^{+/ o}$ parabionts was similar to the blood, with extremely low (<5\%) levels of 
A Non-competitive parabiosis

Competitive parabiosis

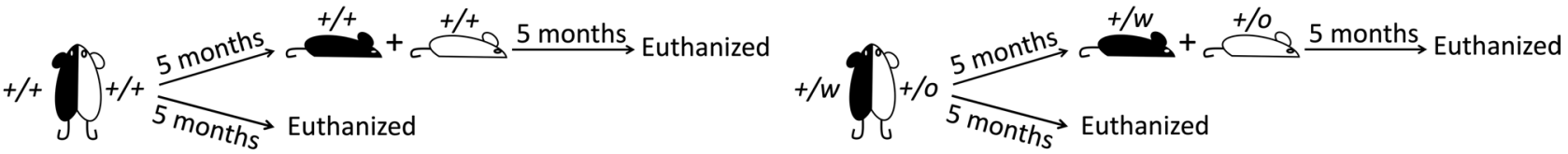
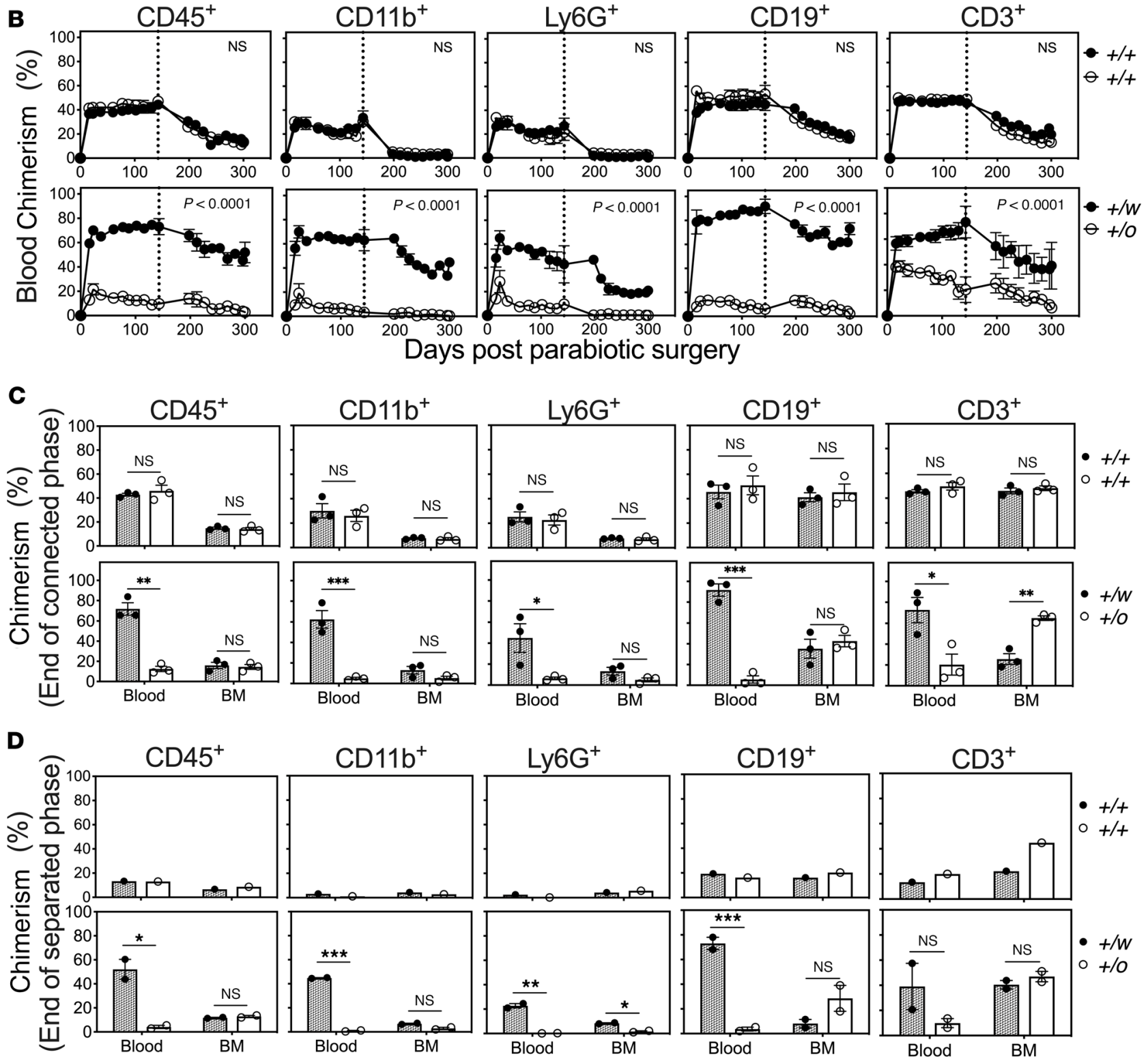

E
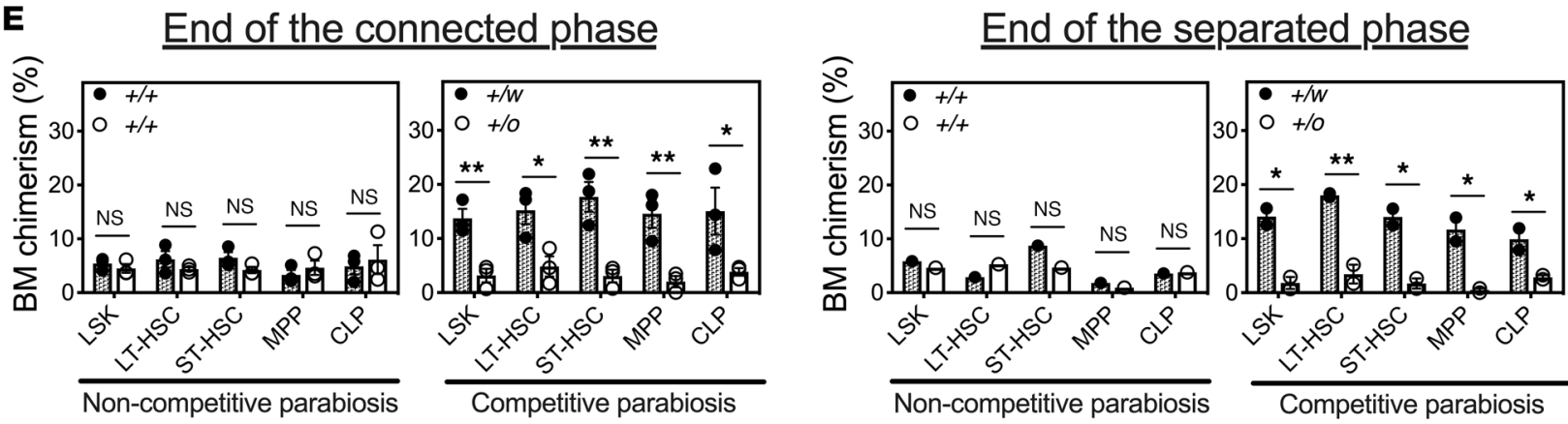
Figure 2. Durable low-level Cxcr4 ${ }^{+/ 0}$ HSC chimerism in BM supports a high level of blood leukocyte chimerism in Cxcr4 ${ }^{+/ w}$ mice. (A) Experimental design. To track leukocytes, each parabiont has a different congenic CD45 background from its partner (CD45.2 or CD45.1/.2). +/o, CxCr4 ${ }^{+/ 0} ;+/ \mathrm{w}$, Cxcr4 $4^{+/ w} ;+/+$, $\mathrm{CxCr}^{+/+}$. (B-D) Mature leukocyte chimerism analysis in blood and BM. Leukocyte subsets are defined by immunophenotype at the top of each column of panels. Symbols for the genotypes of each parabiont are defined at the right. Top row of graphs in each panel: data from $\mathrm{CxCr}^{+/+} / \mathrm{CxCr}^{+/+}$parabiosis; bottom row of graphs in each panel: data from $\mathrm{CxCr}_{4}^{+/ 0} / \mathrm{CxCr}^{+/ / w}$ parabiosis. (B) Time course in blood. Vertical dashed lines indicate the time when the parabiotic mice were separated. (C and D) BM chimerism 5 months after parabiotic surgery, the time of separation of the parabionts (C), or 5 months after separation of the parabionts (D). Blood chimerism at the same 2 time points is reproduced in $\mathbf{C}$ and $\mathbf{D}$ from $\mathbf{B}$ for direct comparison. (E) HSC and HPC analysis in BM. The time of BM analysis is indicated at the top of each panel, and the symbols for each parabiont genotype are defined at the top left within each panel. LSK, lineage $-S c a-1^{+} \mathrm{C}-\mathrm{Kit}^{+} \mathrm{BM}$ cells; LT-and ST-HSC, long-term and short-term hematopoietic stem cells; MPP, multipotential progenitor cells; CLP, common lymphoid precursor cells. (B-E) Data are the summary of all parabiotic pairs created and are presented as the percentage (mean \pm SEM)

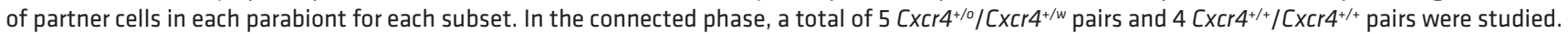
Of these, 3 pairs were terminated in each group for BM analysis at the end of the connected phase, leaving $2 \mathrm{CxCr}^{+/ 0} / \mathrm{CxCr}^{4^{+/ \omega}}$ pairs and $1 \mathrm{CxCr} 4^{+/+} / \mathrm{CxCr} 4^{+/+}$ pair for study in the separated phase. $C x \mathrm{Cr}^{+/ 0} / \mathrm{CxCr}^{+/ w}$ data are combined from parabiotic pairs that were differentially marked with $\mathrm{CD} 45$ isoforms both

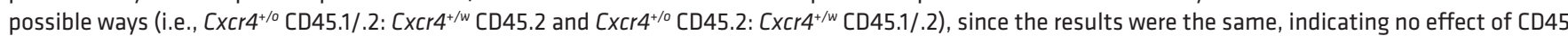
polymorphism. ${ }^{*} P<0.05 ;{ }^{* *} P<0.01 ;{ }^{* *} P<0.005$. Single comparisons, Student's $t$ test; multiple comparisons, 2-way ANOVA.

myeloid chimerism (Figure 2C, bottom). BM Cxcr4 $4^{+/ w} \mathrm{~B}$ cell and T cell chimerism was $40 \%$ and $60 \%$ for the $\mathrm{Cxcr}^{+/ o}$ parabionts, respectively, the same as for $\mathrm{BM} \mathrm{Cxcr} 4^{+/ o} \mathrm{~B}$ cell chimerism in the $\mathrm{Cxcr}^{+/ w}$ parabionts and more than double the level for BM Cxcr $4^{+/ o} \mathrm{~T}$ cell chimerism in the $\mathrm{Cxcr}^{+/ w}$ parabionts (Figure 2C, bottom).

As with the control $\mathrm{Cxcr}^{+/+} / \mathrm{Cxcr}^{+/+}$parabiotic pair, separation of both remaining $\mathrm{Cxcr} 4^{+/ \mathrm{w}} / \mathrm{Cxcr}^{+/ o}$ pairs resulted in a decline in mature $\mathrm{Cxcr} 4^{+/ w}$ leukocyte chimerism in the peripheral blood of the $\mathrm{Cxcr}^{4^{+/ o}}$ parabionts to a new stable level, with myeloid and B cell chimerism declining from approximately $5 \%$ to $0 \%$ and $\mathrm{T}$ cell chimerism from approximately $20 \%$ to approximately $10 \%$ at the end of the separated phase, 5 months after parabiosis (Figure 2B, bottom). However, $\mathrm{CxCr}^{+/ o}$ chimerism in the $\mathrm{CxCr}^{+/ w}$ parabionts was still maintained at the relatively high levels of approximately $40 \%$ myeloid and $60 \%$ lymphoid chimerism after the initial decline (Figure 2B, bottom).

The pattern of BM HSC and HPC chimerism at the end of the separated phase was similar to the pattern at the end of the connected phase, with approximately $15 \% \mathrm{Cxcr}^{+/ o}$ chimerism in the $\mathrm{Cxcr} 4^{+/ w}$ parabiont and approximately $3 \% \mathrm{Cxcr}^{+/ w}$ chimerism in the $\mathrm{Cxcr}^{+/ o}$ parabiont (Figure 2E). The BM myeloid cell chimerism pattern was also similar in the 2 phases (Figure 2D, bottom). The BM lymphoid chimerism pattern at the end of the separated phase was slightly different from the pattern at the end of the connected phase, with $\mathrm{Cxcr}^{+/ o}$ chimerism in the $\mathrm{Cxcr}^{+/ w}$ parabiont of approximately $10 \%$ and $45 \%$ for B and $\mathrm{T}$ cells, respectively, and $\mathrm{Cxcr}^{+/ w}$ chimerism in the $\mathrm{Cxcr}^{+/ o}$ parabiont of approximately $35 \%$ and $50 \%$ for $\mathrm{B}$ and $\mathrm{T}$ cells, respectively (Figure 2D, bottom).

Thus, compared with the isogenic $\mathrm{Cxcr}^{+/+} / \mathrm{Cxcr}^{+/+}$control parabiotic mice, in which there was no difference in chimerism levels between each parabiont in any leukocyte subset in either BM or blood and in either the connected or separated phase, chimerism levels in paired parabionts were strongly skewed by $\mathrm{Cxcr}^{+/ w} / \mathrm{Cxcr}^{+/ o}$ competition in parabiotic mice in all subsets analyzed in both compartments and both phases. Owing to the stress placed on mice by parabiotic life, we limited the analysis to these 2 pairs, which gave results consistent with the results of $\mathrm{Cxcr} 4^{+/ w} / \mathrm{Cxcr}^{+/ o}$ competitive transplantation experiments in both irradiated and unconditioned congenic recipients and with published reports for isogenic $\mathrm{Cxcr} 4^{+++} / \mathrm{Cxcr}^{+/+}$ parabiotic mice $(15,19,22,23)$. The likelihood that the other 2 isogenic control pairings $\left(\mathrm{Cxcr} 4^{+/ 0} / \mathrm{Cxcr}^{+/ 0}\right.$, $\mathrm{Cxcr} 4^{+/ w} / \mathrm{Cxcr}^{+/ w}$ ) would give different skewed results is very low.

\section{Discussion}

The present study demonstrates that $C x c r 4$ haploinsufficiency enhances hematopoietic reconstitution in the setting of both BM transplantation and parabiosis in mice and suggests that it may have been a contributory genetic mechanism, but possibly not the sole genetic mechanism, underlying the remarkable cure of WHIM patient WHIM-09. The results confirm and extend our previous results testing the effects of $C x c r 4$ haploinsufficiency on HSC engraftment in mice using less stringent conditions that were limited to transplantation $(15,19)$. Most importantly, the results indicate that even low-level engraftment of Cxcr4-haploinsufficient HSCs in BM is sufficient to correct leukopenia in WHIM mice, which has important implications for gene therapy strategies in the disease.

Precise delineation of the hematologic cure mechanism for WHIM-09 requires a minimum of 2 elements: a mechanism for silencing the hyperactive WHIM allele in an HSC and a mechanism for replacing WHIM hematopoiesis with nonleukemic clonal expansion of the original chromothriptic HSC. The chro- 
mothriptic event provides a unifying genetic mechanism for both elements. Chromothriptic deletion on 1 copy of chromosome 2 is a straightforward and obvious explanation for WHIM allele silencing in HSCs and the myeloid lineage. Moreover, the myeloid selectivity of the chromothriptic event not only reveals that CXCR4 haploinsufficiency in the myeloid lineage is a viable genetic state, but also that myeloid expression of the WHIM mutation may be a dominant factor driving the main clinical manifestations of the disease. However, identifying the gene(s) that contributed to clonal expansion of the original chromothriptic HSC is not straightforward, because all combinations of the 164 genes deleted in the original chromothriptic HSC must be considered as potential candidates, a puzzle that cannot be solved in the patient. Defining the relevant gene(s) is of particular importance for strategies to cure WHIM syndrome but also has general importance, because this information may provide a target for development of interventions to facilitate HSC engraftment after any transplantation application, with the goal of limiting or even eliminating the toxicity of current conditioning methods.

The challenge to identifying the relevant engraftment-enhancing gene(s) lies not only in the large number of genetic possibilities but also in the fact that the chromothriptic event occurred in vivo presumably in an HSC niche, a setting that we cannot precisely mimic experimentally. Nevertheless, any translational engraftment-enhancing method based on the underlying selective mechanism in WHIM-09 would involve transplantation, so we focused initially on mouse models of transplantation. We tested the most parsimonious hypothesis, that deletion of the WHIM allele by itself is sufficient to explain the selective advantage of the chromothriptic HSC and the complete myeloid conversion in WHIM-09. This hypothesis is consistent with the known antiproliferative effect of CXCR4 signaling in HSCs (5), and indeed, we previously demonstrated that $\mathrm{Cxcr}^{+/ o} \mathrm{HSCs}$ are hyperproliferative in vivo relative to $\mathrm{Cxcr} 4^{+/ w} \mathrm{HSCs}$ when competitively transplanted into lethally irradiated mice (19). Our previously published $(15,19)$ and current results show that both in lethally irradiated wild-type recipient mice and nonconditioned unmanipulated $C x c r 4^{+/ w}$ WHIM model recipient mice, Cxcr4 haploinsufficiency dramatically enhances hematopoietic reconstitution of the B cell and myeloid lineages. Remarkably, in irradiated recipients as few as $1 \%$ of $C \mathrm{Cr}^{4}{ }^{+/ o}$ donor HSCs $(\sim 5 \mathrm{HSC}$ transferred per mouse) could successfully compete against $99 \%$ of $C x c r 4^{+/ w}$ donor HSCs to establish $>20 \%$ long-term $\mathrm{Cxcr}^{+/ o} \mathrm{HSC}$ and HPC chimerism in $\mathrm{BM}$ and $>50 \%$ stable $\mathrm{Cxcr} 4^{+/ o}$ myeloid and B cell chimerism in the blood. As expected, as the ratio of donor $C x c r 4^{+/ o} \mathrm{BM}$ cells increased, the proportion of $C x c r 4^{+/ o}$ hematopoiesis increased. Since the $C x c r 4^{+/ w}$ genotype favors skewing of mature hematopoietic cells to the BM (the key feature of myelokathexis) and the $C x c r 4^{+/ o}$ does not, the total number of cells in the circulation for each subset should decline as the donor $C x c r 4^{+/ w} / C x c r 4^{+/ o}$ dose ratio increases, which is what we observed. While the potency of Cxcr4 haploinsufficiency revealed by these new graded dose-response studies is impressive, the results in transplanted lethally irradiated recipient mice clearly indicate that a single $C x c r 4^{+/ o} \mathrm{HSC}$ is unable to compete with a great excess of $\mathrm{CxCr}^{+/ w} \mathrm{HSC}$ to fully repopulate both the HSC pool and the entire myeloid lineage, which is what happened in WHIM-09 (15). Previously, we performed Cxcr4 ${ }^{+/ o} \mathrm{BM}$ transplantation in completely unconditioned $C x c r 4^{+/ w}$ recipient mice, an experiment in which the donor $C x c r 4^{+/ o} \mathrm{HSCs}$, but not the recipient mice and recipient $C x c r 4^{+/ w} \mathrm{HSCs}$, were manipulated ex vivo and demonstrated that $C x c r 4^{+/ o} \mathrm{BM}$ donor cells ( $\sim 5000$ donor $C x c r 4^{+/ o} \mathrm{HSCs}$ ) could establish approximately $70 \%$ donor myeloid and B cell chimerism in the blood (19). This was enough chimerism to correct leukopenia in $C x c r 4^{+/ w}$ mice but not enough to repopulate the entire recipient $C x c r 4^{+/ w}$ myeloid lineage, suggesting that the $C x c r 4^{+/ o} \mathrm{HSCs}$ transplanted in this experiment are less potent than the chromothriptic HSC in WHIM-09.

To obviate potential artifacts of the transplantation process on engraftment, we surgically created parabiotic $\mathrm{Cxcr}^{+/ o} / \mathrm{Cxcr}^{+/ w}$ mouse pairs. In this parabiotic system, hematopoietic cells, including HSCs and HPCs in the peripheral blood, could migrate to each parabiont, and some of the HSCs and HPCs in the peripheral blood could home to BM of the opposite parabiont, resulting in HSC chimerism without any in vitro manipulation. The results showed durable $80 \%-100 \% \mathrm{CxCr}^{+/ 1}$ myeloid and $\mathrm{B}$ and $\mathrm{T}$ cell chimerism in the blood and $15 \% \mathrm{Cxcr}^{+/ o} \mathrm{HSC}$ chimerism in BM of the $\mathrm{Cxcr}^{+/ w}$ parabiont, which was maintained for 5 months after separation from the $\mathrm{Cxcr}^{+/ o}$ parabiont when the experiment was terminated. This was much higher chimerism than that observed in the $C x c r 4^{+/ o}$ parabiont partner and the wild-type parabiotic control pairs. However, this experiment also indicated that $C x c r 4$ haploinsufficiency alone is not sufficient to replace the entire population of $\mathrm{CxCr}^{+/ w} \mathrm{HSCs}$ and entire $\mathrm{Cxcr} 4^{+/ w}$ myeloid lineage. A possible difference between this experiment and the chromothriptic HSC in WHIM-09, other than the additional 163 genes deleted in WHIM-09, could be related to different environmental conditions for the $\mathrm{Cxcr}^{+/ o} \mathrm{HSC}$ in the BM of the $C x \mathrm{cr} 4^{+/ w}$ parabiont, which must have transited through the blood from the $\mathrm{Cxcr}^{+/ o}$ parabiont, relative to the chromothriptic HSC in WHIM- 
09, which may have been resident in the BM. In this regard, it has been reported that HSCs in the circulation are in general less totipotent than those in the $\operatorname{BM}(22,24,25)$.

We previously suggested that hematopoietic reconstitution may be enhanced by Cxcr4 haploinsufficiency in donor HSCs because of 2 mechanisms: enhanced proliferation of HSCs and enhanced release of mature leukocytes from BM. The former mechanism is most evident in the lethally irradiated recipient model, where there is maximum availability of stem cell niches and a massive excess of $\mathrm{CxCr}^{+/ o} \mathrm{HSCs}$ over $\mathrm{Cxcr} 4^{+/ w} \mathrm{HSCs}$ in $\mathrm{BM}$ at equilibrium, overcoming a BM homing advantage conferred by the WHIM allele early on. In contrast, in unconditioned $\mathrm{Cxcr}^{4 / \mathrm{w}}$ recipient mice and parabiotic mice, niche availability is minimal, which may explain why the frequencies of $\mathrm{Cxcr}^{+/ o}$ and $\mathrm{Cxcr}^{+/ w} \mathrm{HSCs}$ in BM at equilibrium were much less skewed in the parabiosis experiments and not different in the unconditioned $C x c r 4^{+/ w}$ recipients. Thus, in these 2 models, $C x c r 4^{+/ o}$ enhancement of hematopoietic reconstitution in the blood appears to be driven by enhanced release of $\mathrm{Cxcr}^{+/ 0}$ mature leukocytes from BM, probably supported by enhanced proliferation of engrafted $C x c r 4^{+/ o} \mathrm{HSCs}$. In the 2 transplantation models, a large number of donor HSCs were transplanted all at once, whereas in parabiosis, the number of the donor HSCs is limited by the frequency of HSCs in the blood (about 1 in every 100,000 nucleated blood cells, ref. 26) and the transplantation process continues until the 2 parabionts are separated. Thus, in this model $\mathrm{CxCr}^{+/ \mathrm{o}}$ enhancement of hematopoietic reconstitution in the blood may be driven more by enhanced mature leukocyte release from BM with a contribution from enhanced HSC engraftment in BM. Taken together, our results suggest that the increased proliferative potential of $\mathrm{Cxcr}^{+/ \mathrm{o}} \mathrm{HSCs}$ over $\mathrm{Cxcr} 4^{+/ \mathrm{w}} \mathrm{HSCs}$ may only become manifest when stem cell niches are present in great excess over available donor HSCs or when more mature leukocytes are needed for an immune response. In contrast, the foundational chromothriptic HSC in WHIM-09 was able to replace the entire WHIM HSC population.

In conclusion, our study of WHIM-09 and efforts to model her cure in mice suggest that other factors besides chromothriptic deletion of the WHIM allele may have played a significant role in conversion of the myeloid lineage. Future work will be needed to prioritize the 163 other genes for analysis of effects of haploinsufficiency on hematopoietic reconstitution activity.

Although complete Cxcr4 deficiency is embryonic lethal in mice (27), and CXCR4 gain-of-function mutations cause WHIM syndrome and are associated with worse outcomes and poorer responses to therapy in patients with Waldenstrom's macroglobulinemia (28), the only phenotype we have identified to date that defines Cxcr4 hemizygosity as a haploinsufficient state is enhanced hematopoietic reconstitution activity in the context of transplantation. Importantly, our combined results in all 3 competitive transplantation models show that even low-level $\mathrm{Cxcr}^{+/ o} \mathrm{HSC}$ and HPC engraftment is sufficient to correct leukopenia in WHIM model mice, suggesting that to correct leukopenia and cure WHIM patients only low-level engraftment of donor CXCR4-haploinsufficient HSCs may be necessary. Further, our parabiosis experiments indicated that $\mathrm{CxCr}^{+/ o} \mathrm{HSC}$ from the circulation also have enhanced hematopoietic reconstitution activity in blood after homing to the BM of the WHIM recipient, suggesting the feasibility of gene therapy for WHIM syndrome by deletion of the CXCR4 disease allele in HSCs collected from the blood of WHIM patients. Efforts to test this concept using gene editing are currently underway in our laboratory. More broadly, the results also imply that CXCR4 knockdown may be a useful general genetic adjuvant strategy to enhance engraftment of donor HSCs and spare patients from genotoxic conditioning in the setting of syngeneic and possibly allogeneic transplantation.

\section{Methods}

Mice. Mouse strains $\mathrm{CxCr}^{+/ o}$ on CD45.1 or CD45.2 or a heterozygous CD45.1/CD45.2 background and $\mathrm{CxCr}^{+/ w}$ on CD45.2 or a heterozygous CD45.1/CD45.2 background have been previously described (15, 19). All the wild-type mice used were the littermates of $C x c r 4^{+/ o}$ or $C x c r 4^{+/ w}$ mice. The mice were kept in a specific pathogen-free facility at NIH and were 6 to 8 weeks old at the time of transplantation.

Transplantation experiments. Total BM cells (from a single donor strain or as a mixture of cells from 2 donor strains) were transferred via tail vein injection into recipient mice that had undergone lethal irradiation (9 Gy) 8 hours prior to transplantation. The donor ratio for mixed BM cells in each competitive transplantation was determined by flow cytometric analysis of CD45 markers. In each experiment, donors and recipients were sex matched. Results were similar in both male and female mice. Irradiated mice were given water containing neomycin for 4 weeks after irradiation to reduce mortality from infection.

Parabiosis. Each pair of female mice was cohoused before surgery for 2 weeks, beginning at 6 weeks of age. Pairs were surgically connected as previously described $(23,29)$. The mice were anesthetized to full 
muscle relaxation with xylazine and ketamine $\mathrm{HCl}(1.67 \mathrm{mg}$ per $10 \mathrm{~g}$ of body weight) by intraperitoneal injection. After shaving the corresponding lateral aspects of each mouse, matching skin incisions were made, starting $0.5 \mathrm{~cm}$ distal to the elbow to $0.5 \mathrm{~cm}$ below (distal to) the knee joint, and the subcutaneous fascia was bluntly dissected to create about 0.5 to $1.0 \mathrm{~cm}$ of free skin. The olecranon and knee joints were connected by a single 2-0 silk suture and tie, and the skin of the 2 animals was connected with a continuous absorbable 5-0 Vicryl suture material. The parabiotic mice were treated with trimethoprim/sulfamethoxazole-containing drinking water for at least 10 days after surgery to prevent bacterial infections and given ibuprofen-containing drinking water for 48 hours to limit postsurgical pain. In some experiments, parabiotic mice were surgically separated by a reversal of the above procedure.

To track leukocytes, each parabiont in each parabiotic pair had a different CD45 background (CD45.2 or CD45.1/.2). To exclude possible effects of CD45 background on the parabiosis results, we switched the CD45.2 and CD45.1/.2 background in $\mathrm{CxCr}_{4}^{+/ o}, \mathrm{CxCr} 4^{+/ w}$, and $\mathrm{CxCr}^{+/+}$mice in a separate experiment, which showed similar results. Therefore, we combined the experimental results in Figure 2.

Blood cell counts. Blood was collected from mandibular veins of recipient mice using EDTA as anticoagulant (BD). Total leukocyte counts were measured with a Cellometer Auto 2000 Cell Viability Counter (Nexcelom Bioscience). Absolute leukocyte subset counts and frequencies were quantitated by flow cytometry.

Flow cytometry analysis. For leukocyte subset analysis of the cells from blood and BM, $100 \mu$ of mouse blood was collected and incubated for 10 minutes with $2 \mu \mathrm{l}$ of Fc block (BioLegend) prior to incubation with specific antibodies (see below) on ice for 30 minutes. The monoclonal antibodies used were CD45.1-PECy7 and CD45.2-eFlour450 (catalog no. 25-0453-82 and no. 48-0454-82, eBioscience) and Ly6G-APCCy7, CD11b-PerCP-Cy5.5, CD19-FITC, and CD3-APC (catalog no. 127624, 101228, 115506, and 100312, Biolegend). Erythrocytes were lysed with $2 \mathrm{ml} \mathrm{ACK}$ lysis buffer (Quality Biologicals Inc.) for 2 minutes at room temperature before centrifugation. Cells were then washed twice with FACS buffer, fixed with 1\% paraformaldehyde, stored on ice, and analyzed using an LSRII FACS cytometer (BD Biosciences) and FlowJo software (TreeStar Inc.). A similar procedure was followed for staining BM cells isolated from femurs and tibiae. For HSC and HPC analysis, BM cells were isolated from femurs and tibiae. $8 \times 10^{6}$ cells in $80 \mu \mathrm{l}$ of FACS buffer were incubated for 20 minutes with biotinylated anti-CD34 mAb (clone RAM from eBioscience), washed once, and resuspended in $70 \mu \mathrm{l}$ of FACS buffer. Then, the cells were stained with the following antibodies: Lin-FITC (FITC-conjugated antibodies against lineage markers, including B220, CD3, CD4, CD8, CD11b, Gr1, and Ter119), Sca1-APC-Cy7, c-Kit-APC, Flt3-PE-Cy5, IL7ra-Violet 605, and streptavidin-PE (catalog no. 133302, 108126, 105812, 135312, 135025, and 405203, Biolegend). The HSCs and HPCs were defined as follows: long-term HSC (LT-HSC: $\mathrm{Lin}^{-} \mathrm{Sca}^{+}{ }^{+} \mathrm{C}-\mathrm{Kit}^{+} \mathrm{CD} 34^{-} \mathrm{Flt}^{-}$), short-term HSC (ST-HSC: $\mathrm{Lin}^{-} \mathrm{Sca}^{+}{ }^{+} \mathrm{C}-\mathrm{Kit}^{+} \mathrm{CD} 34^{+} \mathrm{Flt3} 3^{-}$), common lymphoid progenitors (CLP: IL7 $\mathrm{ra}^{+} \mathrm{Lin}^{-}$ Sca $1^{10} \mathrm{C}-\mathrm{Kit}^{1 \mathrm{lo}}$ ), multipotent progenitors (MPP: $\mathrm{Lin}^{-} \mathrm{Sca} 1^{+} \mathrm{C}-\mathrm{Kit}^{+} \mathrm{CD} 34^{+} \mathrm{Flt} 3^{+}$), and LSK $\left(\mathrm{Lin}^{-} \mathrm{Sca}^{+} \mathrm{C}-\mathrm{Kit}^{+}\right.$).

Statistics. Data are expressed as mean \pm SEM. Two-tailed Student's $t$ test for single comparisons and 2-way ANOVA for multiple comparisons were used. $P$ values of less than 0.05 were considered significant.

Study approval. All animal experiments were performed using an National Institute of Allergy and Infectious Diseases Animal Care and Use Committee-approved protocol in approved and certified facilities.

\section{Author contributions}

Experimental design was provided by JLG, DHM, and PMM. Generation of experimental data was provided by JLG, AOA, AP, KB, EY, MS, AY, and QL, with supervision and analysis by JLG and PMM. JLG and PMM were principally responsible for writing the manuscript.

\section{Acknowledgments}

This work was supported by the Division of Intramural Research of the National Institute of Allergy and Infectious Diseases, NIH. The authors thank Karl Balabanian and Francoise Bachelerie for the gift of WHIM model mouse strain $C x c r 4^{+/ 1013}$.

Address correspondence to: Philip M. Murphy, Building 10, Room 11N113, 10 Center Drive, NIH, Bethesda, Maryland 20892, USA. Phone: 301.496.8616; Email: pmm@nih.gov.

1. Hernandez PA, et al. Mutations in the chemokine receptor gene CXCR4 are associated with WHIM syndrome, a combined immunodeficiency disease. Nat Genet. 2003;34(1):70-74. 
2. Heusinkveld LE, et al. Pathogenesis, diagnosis and therapeutic strategies in WHIM syndrome immunodeficiency. Expert Opin Orphan Drugs. 2017;5(10):813-825.

3. Dar A, Kollet O, Lapidot T. Mutual, reciprocal SDF-1/CXCR4 interactions between hematopoietic and bone marrow stromal cells regulate human stem cell migration and development in NOD/SCID chimeric mice. Exp Hematol. 2006;34(8):967-975.

4. Broxmeyer HE, et al. Rapid mobilization of murine and human hematopoietic stem and progenitor cells with AMD3100, a CXCR4 antagonist. J Exp Med. 2005;201(8):1307-1318.

5. Nie Y, Han YC, Zou YR. CXCR4 is required for the quiescence of primitive hematopoietic cells. J Exp Med. 2008;205(4):777-783.

6. Sugiyama T, Kohara H, Noda M, Nagasawa T. Maintenance of the hematopoietic stem cell pool by CXCL12-CXCR4 chemokine signaling in bone marrow stromal cell niches. Immunity. 2006;25(6):977-988.

7. Kawai T, et al. WHIM syndrome myelokathexis reproduced in the NOD/SCID mouse xenotransplant model engrafted with healthy human stem cells transduced with C-terminus-truncated CXCR4. Blood. 2007;109(1):78-84.

8. Gorlin RJ, Gelb B, Diaz GA, Lofsness KG, Pittelkow MR, Fenyk JR. WHIM syndrome, an autosomal dominant disorder: clinical, hematological, and molecular studies. Am J Med Genet. 2000;91(5):368-376.

9. McDermott DH, et al. AMD3100 is a potent antagonist at CXCR4(R334X), a hyperfunctional mutant chemokine receptor and cause of WHIM syndrome. J Cell Mol Med. 2011;15(10):2071-2081.

10. Beaussant Cohen S, et al. Description and outcome of a cohort of 8 patients with WHIM syndrome from the French Severe Chronic Neutropenia Registry. Orphanet J Rare Dis. 2012;7:71.

11. Dotta L, Tassone L, Badolato R. Clinical and genetic features of Warts, Hypogammaglobulinemia, Infections and Myelokathexis (WHIM) syndrome. Curr Mol Med. 2011;11(4):317-325.

12. Latger-Cannard V, Bensoussan D, Bordigoni P. The WHIM syndrome shows a peculiar dysgranulopoiesis: myelokathexis. $B r J$ Haematol. 2006;132(6):669.

13. Palm MD, Tyring SK, Rady PL, Tharp MD. Human papillomavirus typing of verrucae in a patient with WHIM syndrome. Arch Dermatol. 2010;146(8):931-932.

14. Zuelzer WW. "Myelokathexis"--a New Form of Chronic Granulocytopenia. Report of a Case. N Engl J Med. 1964;270:699-704.

15. McDermott DH, et al. Chromothriptic cure of WHIM syndrome. Cell. 2015;160(4):686-699.

16. Stephens PJ, et al. Massive genomic rearrangement acquired in a single catastrophic event during cancer development. Cell. 2011;144(1):27-40.

17. Jones MJ, Jallepalli PV. Chromothripsis: chromosomes in crisis. Dev Cell. 2012;23(5):908-917.

18. Kloosterman WP, et al. Chromothripsis as a mechanism driving complex de novo structural rearrangements in the germline. Hum Mol Genet. 2011;20(10):1916-1924.

19. Gao JL, et al. Cxcr4-haploinsufficient bone marrow transplantation corrects leukopenia in an unconditioned WHIM syndrome model. J Clin Invest. 2018;128(8):3312-3318.

20. Mayle A, Luo M, Jeong M, Goodell MA. Flow cytometry analysis of murine hematopoietic stem cells. Cytometry A. 2013;83(1):27-37.

21. Gibney BC, et al. Cross-circulation and cell distribution kinetics in parabiotic mice. J Cell Physiol. 2012;227(2):821-828.

22. Abkowitz JL, Robinson AE, Kale S, Long MW, Chen J. Mobilization of hematopoietic stem cells during homeostasis and after cytokine exposure. Blood. 2003;102(4):1249-1253.

23. Wright DE, Wagers AJ, Gulati AP, Johnson FL, Weissman IL. Physiological migration of hematopoietic stem and progenitor cells. Science. 2001;294(5548):1933-1936.

24. McKinney-Freeman S, Goodell MA. Circulating hematopoietic stem cells do not efficiently home to bone marrow during homeostasis. Exp Hematol. 2004;32(9):868-876.

25. Morita Y, Iseki A, Okamura S, Suzuki S, Nakauchi H, Ema H. Functional characterization of hematopoietic stem cells in the spleen. Exp Hematol. 2011;39(3):351-359.e3.

26. Ng YY, Baert MR, de Haas EF, Pike-Overzet K, Staal FJ. Isolation of human and mouse hematopoietic stem cells. Methods Mol Biol. 2009;506:13-21.

27. Ma Q, et al. Impaired B-lymphopoiesis, myelopoiesis, and derailed cerebellar neuron migration in CXCR4- and SDF-1-deficient mice. Proc Natl Acad Sci USA. 1998;95(16):9448-9453.

28. Hunter ZR, et al. The genomic landscape of Waldenstrom macroglobulinemia is characterized by highly recurring MYD88 and WHIM-like CXCR4 mutations, and small somatic deletions associated with B-cell lymphomagenesis. Blood. 2014;123(11):1637-1646.

29. Lee SH, et al. Mannose receptor high, M2 dermal macrophages mediate nonhealing Leishmania major infection in a Th1 immune environment. J Exp Med. 2018;215(1):357-375. 